\title{
EDITORIAL
}

For reprint orders, please contact: reprints@futuremedicine.com

\section{Bevacizumab therapy for glioblastoma: a passionate discussion}

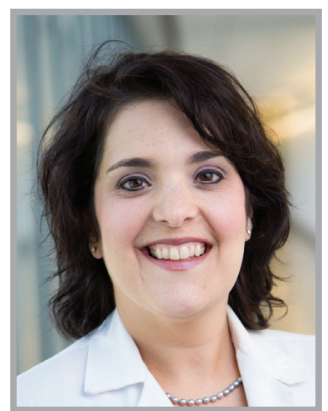

Annick Desjardins ${ }^{1}$

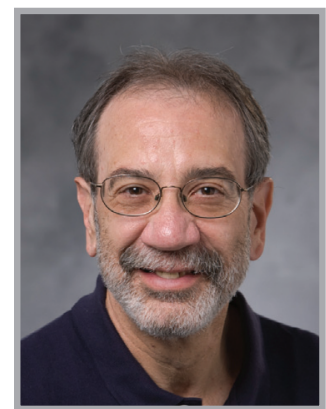

Henry S Friedman*1

\author{
"Bevacizumab given with \\ irinotecan was initially \\ shown ... to produce \\ extraordinary radiographic \\ and clinical improvements \\ in patients with glioblastoma \\ multiforme refractory to \\ many prior treatments."
}

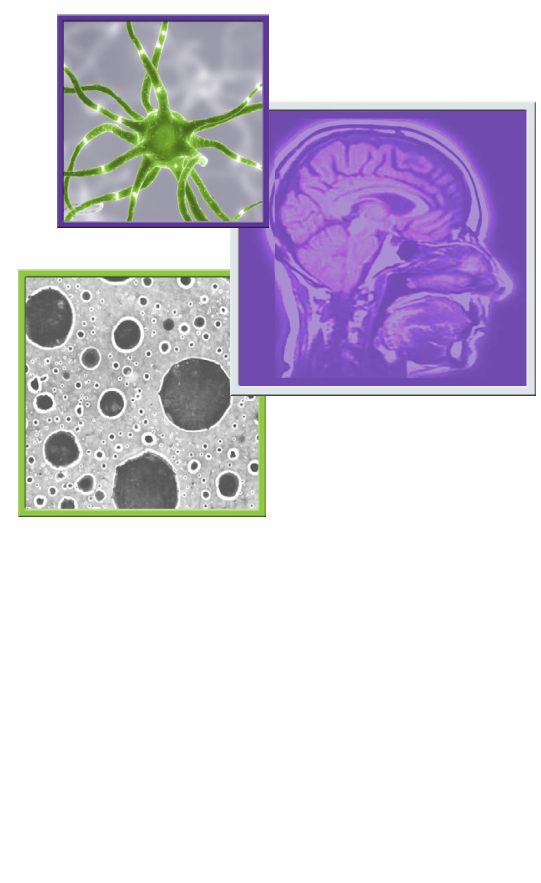

Few, if any, topics have ignited the passions of the global neuro-oncology world more than the role of bevacizumab in the treatment of patients with glioblastoma mulitforme (GB). From its relatively recent anecdotal [1] and single institution trials of recurrent disease $[2,3]$ to the multi-institutional study that led to US FDA approval of bevacizumab in the USA for recurrent GBM [4], to single-arm trials of newly diagnosed patients $[5,6]$ and finally to two randomized Phase III trials in newly diagnosed patients presented at the 2013 meeting of the American Society of Clinical Oncology [7,8], strong passions have been ubiquitous and at this point predictable and anticipated.

Why then has a pharmaceutical agent so inflamed the world's neuro-oncologists and related specialists? The answers lie, as in all controversies, with the disparate interpretation of the available data held by the leaders and their followers in this field.

Certain basic facts are immutable and do not appear to be controversial. Bevacizumab given with irinotecan was initially shown by Stark-Vance to produce extraordinary radiographic and clinical improvements in patients with GBM refractory to many prior treatments [1]. Vredenburgh et al. subsequently demonstrated prodigious radiographic and clinical responses in single-arm clinical trials [2,3], although it became clear that T1-contrasted MRI could prove misleading, requiring the consideration of fluid-attenuated inversion recovery imaging, a concept subsequently formalized into the modified Revised Assessment in Neuro-Oncology (RANO) criteria [9].

The BRAIN Trial [4], incorporating this important observation, confirmed the response rate of bevacizumab alone or in combination with irinotecan, leading to FDA approval of bevacizumab in patients with recurrent GBM. This became a major point of contention in Europe, and in some, but fewer, US institutions, because a true comparative control group was not included. Nevertheless, it is safe to say that although there will always be skeptics who doubt the benefits of any new agent, bevacizumab does help patients with recurrent disease. However, it certainly does not help all such patients, but currently we do not have any test or genetic profile to reliably differentiate responders from
"... it is safe to say that although there will always be skeptics who doubt the benefits of any new agent, bevacizumab does help patients with recurrent disease." 


\section{"Questions thus have been raised as to whether bevacizumab should be given at first recurrence or at a later recurrence."}

nonresponders. Critics will say that bevacizumab makes it harder to treat patients whose disease progress while receiving it, yet are hard pressed to identify a treatment that is more meaningful. Concerns regarding the patterns of failure, specifically proangiogenic enhancing disease at the initial presentation site $[4,10]$ versus increased proinvasive diffuse disease [11], are frequently voiced with different reports indicating a change versus no change compared with nonbevacizumab-treated patients who demonstrate recurrent tumor. Questions thus have been raised as to whether bevacizumab should be given at first recurrence or at a later recurrence. Proponents of this appear unfazed by the observation that many patients do not receive any therapy as subsequent recurrences are seen.

The issues regarding the treatment of patients with newly diagnosed GBM are even more contentious. The definitive studies are AVAglio and RTOG 0825, which reported at the American Society of Clinical Oncology 2013 meeting results so different and confusing that it led the executive director, Max Wallace, of one of the leading brain tumor advocacy groups in the world, Accelerate Brain Cancer Cure, to ask for a FDA review of both trials [12]. In essence, both groups reported similar overall survival with no differences in bevacizumab- or placebo-treated patients, an observation probably, although not definitively, owing to the crossover of patients on the placebo arm who subsequently received bevacizumab, as well as owing to the vast majority of patients who initially got bevacizumab but discontinued it at recurrence [13]. The progression-free survival in both studies showed similar increases with similar p-values, but owing to statistical design differences, only the AVAglio study considered the increase meaningful. Most disturbing and extremely controversial were the disparate results showing the neurocognitive/quality of life benefits (AVAglio) versus detrimental effects (RTOG 0825) produced by bevacizumab. Howard Fine (New York University Langone), in what we consider to be a truly brilliant summation, addressed these concerns and argued for a third party review of all the data in both trials.

So where are we now? We know that bevacizumab works in the treatment of recurrent GBM; that not all patients derive benefit; it increases progression-free survival in patients with newly diagnosed tumors; it may or may not enhance or worsen neurocognition/quality of life in these patients; and, in our opinion, it will enhance survival in specific cohorts of patients with newly diagnosed and recurrent GBM. Furthermore, although there are those who suggest bevacizumab should be simply used as a salvage therapy, we should note that even on a clinical trial with a mandatory crossover (RTOG 0825) less than half the patients ever received bevacizumab at tumor progression. One can assume this will be even lower in patients who were not treated in a clinical trial. It is very easy to criticize work in any field, but we suggest that time is better spent trying to make improvements rather than seek reasons why something others did is of no merit. We eagerly await the FDA reviews of the AVAglio and RTOG 0825 trials.

Financial \& competing interests disclosure HS Friedman is a consultant and speaker for Rochel Genentech. A Desjardins is on the Advisory Board for Roche/Genentech. The authors have no other relevant affiliations or financial involvement with any organization or entity with a financial interest in or financial conflict with the subject matter or materials discussed in the manuscript apart from those disclosed.

No writing assistance was utilized in the production of this manuscript.

\section{References}

1 Stark-Vance V. Bevacizumab and CPT-11 in the treatment of relapsed malignant glioma. Neuro-Oncology 7(3), 369 (2005).

2 Vredenburgh JJ, Desjardins A, Herndon JE 2nd et al. Phase II trial of bevacizumab and irinotecan in recurrent malignant glioma. Clin. Cancer Res. 13(4), 1253-1259 (2007).

3 Vredenburgh JJ, Desjardins A, Herndon JE 2nd et al. Bevacizumab plus irinotecan in recurrent glioblastoma multiforme. J. Clin. Oncol. 25(30), 4722-4729 (2007).
4 Friedman HS, Prados MD, Wen PY et al. Bevacizumab alone and in combination with irinotecan in recurrent glioblastoma. J. Clin. Oncol. 27(28), 4733-4740 (2009).

5 Lai A, Tran A, Nghiemphu PL et al. Phase II study of bevacizumab plus temozolomide during and after radiation therapy for patients with newly diagnosed glioblastoma multiforme. J. Clin. Oncol. 29(2), 142-148 (2011).

6 Vredenburgh JJ, Desjardins A, Kirkpatrick JP et al. Addition of bevacizumab to standard radiation therapy and daily temozolomide is associated with minimal toxicity in newly diagnosed glioblastoma multiforme. Int J. Radiat. Oncol. Biol. Phys. 82(1), 58-66 (2012).

7 Wick W, Cloughesy TF, Nishikawa R et al. Tumor response based on adapted Macdonald criteria and assessment of pseudoprogression (PsPD) in the Phase III AVAglio trial of bevacizumab (Bv) plus temozolomide (T) plus radiotherapy (RT) in newly diagnosed 
glioblastoma (GBM). J. Clin. Oncol. 31(15S), $114 S$ (2013).

8 Gert MR, Dignam J, Won M et al. Phase III double-blind placebo-controlled trial evaluating bevacizumab (Bev) in patients (Pts) with newly diagnosed glioblastoma (GBM). J. Clin. Oncol. 31(15S), 5S (2013).

9 Wen PY, Macdonald DR, Reardon DA et al. Updated response assessment criteria for high-grade gliomas: response assessment in neuro-oncology working group. J. Clin. Oncol. 28(11), 1963-1972 (2010).

10

Pope WB, Xia Q, Paton VE et al. Patterns of progression in patients with recurrent glioblastoma treated with bevacizumab. Neurology 76(5), 432-437 (2011).

11 Norden AD, Young GS, Setayesh K et al. Bevacizumab for recurrent malignant gliomas: efficacy, toxicity, and patterns of recurrence. Neurology 70(10), 779-787 (2008).

12 A comparison of study designs, between RTOG 0825 and Genentech's AVAglio. Cancer Lett. 39(24) 1-13 (2013).

13 Reardon DA, Herndon JE 2nd, Peters KB et al. Bevacizumab continuation beyond initial bevacizumab progression among recurrent glioblastoma patients. Br. J. Cancer 107(9), 1481-1487 (2012). 